\title{
Correlation between Postural Stability and Functional Mobility in Patients with Knee Osteoarthritis
}

\author{
Arlinda De Hafsari, ${ }^{1}$ Vitriana, ${ }^{2}$ Alwin Tahid $^{3}$ \\ ${ }^{1}$ Faculty of Medicine, Universitas Padjadjaran, ${ }^{2}$ Departmen of Physiology Faculty of Medicine \\ Universitas Padjadjaran, ${ }^{3}$ Department of Physical Medicine and Rehabilitation Faculty of \\ Medicine, Universitas Padjadjaran/Dr. Hasan Sadikin General Hospital Bandung
}

\begin{abstract}
Background: Osteoarthritis $(\mathrm{OA})$ is a degenerative joint disorder caused by progressive erosion of articular cartilage. The joint which is commonly affected is theknee joint. Patient with knee OA has increasing pain with decreasing postural stability, and may cause decrease infunctional mobility of the body. The aim of this study was to analyze the correlation between stability and mobility in patients with knee OA.

Methods: A cross sectional study was performed to 43 patients from the Rheumatology Clinic ofDr. Hasan Sadikin General Hospital, Bandung, during September-October 2013. The 'Timed Up and Go' (TUG) test and stabillometric platform examination were performed to analyze the mobility and stability in subjects. The data were analyzed with Spearman's Correlation.

Results: Four subjects (9.30\%) had normal functional mobility, 31 subjects $(72.09 \%)$ had good mobility and need no help of others in their activities, and 8 subjects $(18.60 \%)$ had problems and could not stand and walk without help. This study also found 39 subjects $(90.69 \%)$ had high risk of fall. The p value were 0.005 for correlation between knee $\mathrm{OA}$ and lateral static postural stability, 0.138 with anteroposterior static stability, 0.067 with dynamic to position of top right stability, 0.344 with dynamic to position of top left stability, 0.384 with dynamic to position of bottom left stability and 0.357 with dynamic to position of bottom right stability.

Conclusions: There is a strong correlation between functional mobility with static postural stability, especially to the lateral position. [AM].2015;2(4):550-4]
\end{abstract}

Keywords: Functional mobility, knee osteoarthritis, postural stability

\section{Introduction}

Osteoarthritis $(\mathrm{OA})$ is also called degenerative joint disorder that iscaused by progressive erosion of articular cartilage..$^{1,2}$ This disorder isthe most common joint disorder in the world. Within western population, this disorder is one of the most common causes of pain, loss of normal function, and disability of adult. The symptoms that appear in this disorder may cause a mobility disruption which can cause limitation of daily activity. The joint commonly affected by OA is theknee joint. ${ }^{3}$ Knee OA has a high rate of incident in the world. ${ }^{4}$ The OA is a most common rheumatic disease in Indonesia. World Health Organization (WHO) data, shows OA cases in Indonesia is $8.1 \%$ from the total population.

The patient with knee OA has increasing pain and weakness that makes thepatient also has decreasing postural stability of the body, which may cause limitation of patient's activity and motivation. ${ }^{5}$ Postural stability is the body foundation to be able to stand and walk freely. The decrease of postural stability in elderly can make a contribution for increase fall risk when doing daily activity. ${ }^{6}$ Knee OA is commonly associated with the postural stability and functional mobility impairment however based on literature concerning this subject, there was not a study which showed the correlation between postural stability and functional mobility in patients of knee 0A. The aim of this study was to analyze the correlation between postural stability and functional mobilityin knee OA.

\section{Methods}

A cross sectional study was carried out to 43 knee OA patients who came to the Rheumatology Clinic of Dr. Hasan Sadikin

Correspondence: Arlinda De Hafsari, Faculty of Medicine, Universitas Padjadjaran, Jalan Raya Bandung-Sumedang Km.21, Jatinangor, Sumedang, Indonesia, Phone: +62 85795915919 Email: arlindadh@yahoo.com 
Table 1 The Result of Mobility of The Subjects using TUG test

\begin{tabular}{ccc}
\hline TUG (seconds) & Interpretation & Total \\
\hline$<10$ & Normal & 4 subjects (9\%) \\
$<20$ & $\begin{array}{c}\text { Good mobility, can walk alone } \\
\text { without help }\end{array}$ & 31 subjects (72\%) \\
$<30$ & $\begin{array}{c}\text { Problem, } \begin{array}{c}\text { can not walk alone, } \\
\text { need help } \\
\text { High risk of falls }\end{array} \\
\text { 814 }\end{array}$ & 8 subjects (19\%) \\
\hline
\end{tabular}

General Hospital, Bandung, during SeptemberOctober 2013. The OA outpatients were defined from patients ofthe Rheumatology Clinic who visited the Rheumatology Clinic for some follow-up or check-up on the day when the examiner came to the clinic. Patients who agreed to be examined were included in this study, whereas the exclusion criterion is the patients who were not able to stand and walk alone without help. The selected patients were examined in the Physical Medicine and Rehabilitation Clinic with the "Timed Up and Go' (TUG) test and stabillometric platform test. The purpose of the TUG test was to identify how long the subject can stand, walk three meters, and back to the first position, and then sit down again. The criteria of TUG test were: $<10$ seconds: Normal, $<20$ seconds: Good mobility, can walk alone without help, $<30$ seconds: Problem, can not walk alone, need help, $\geq 14$ seconds: High risk of falls.

The stabillometric platform test was to identify the body balance of the patient and the time thepatient needed to move the body to lateral and anteroposterior. This test used the stabillometric platform tool which is based on the software program. The collected data were analyzed using Spearman Correlation.

\section{Results}

The result of functional mobility using TUG test can be classified into four classifications. Most of the subjects had high risk of falls and only $9 \%$ had normal TUG test (Table 1 ).

The result of static postural stability was acquired with the stabillometric platform test, and the results were the point of static condition that was describe by $\mathrm{X}$ and $\mathrm{Y}$ point (Table 2). This result shows the body stability of subject whether its position was to lateral or anteroposterior.

After the static postural stability test, thesubjects were also examined for their dynamic postural stability. This test is also available in the same software of stabillometric platform, therefore the direction and the time were set by the software. It caused time limitation to examine these 43 subjects and the data were not obtained completely.

The result of dynamic postural stability was obtained from stabillometric platform test. That result were the range of time that thesubjects needed for performing this test (Table 3).

After the result of postural stability and functional mobility of the subject was obtained, then the examiner identified the correlation between those variables using the Spearman's Correlation (Table 4).

\section{Discussion}

The risk of fall can make a disruption of body balance that was associated with the functional mobility. If the balance was disrupted, the mobility also decrease and can cause a decreasing quality of life. The result

Table 2 Static Postural Stability of Subjects

\begin{tabular}{ccc}
\hline & Static Stability & Total (\%) \\
\hline X Axis (balance to lateral) & Tendency to the right (positive value) & 17 subjects (40\%) \\
& Tendency to the left (negative value) & 23 subjects (53\%) \\
Y Axis (balance to anteroposterior) & Tendency to the front (positive value) & 32 subjects (74\%) \\
& Tendency to the back (negative value) & 8 subjects (19\%) \\
\hline
\end{tabular}


Table 3 Dynamic Postural Stability of Subjects

\begin{tabular}{ccc}
\hline Target in Position & Range of Time (seconds) & Total Subject \\
\hline Target in top right quarter & $<2$ & 8 \\
& $2-3$ & 13 \\
Target in top left quarter & $3-5$ & 4 \\
& $>5$ & 7 \\
Target in bottom left quarter & $<2$ & 11 \\
& $2-3$ & 7 \\
Target in bottom right quarter & $3-5$ & 9 \\
& $>5$ & 4 \\
& $<2$ & 8 \\
& $2-3$ & 12 \\
& $3-5$ & 10 \\
& $>5$ & 4 \\
\hline
\end{tabular}

of this study also found a high risk of fall in the subjects which was around $91 \%$ or about 39 subjects. The knee OA associated with the presence of fall may cause worse functional mobility. ${ }^{7}$ In 2008, Shaheen et al. ${ }^{8}$ published that patients with knee OA have a worse balance, both static and dynamic, and also worse functional mobility. The impairment of balance is also associated with pain suffered by the patients. Most of thesubjects have a tendency to put the body in bending forward position (74\%) (Table 2), this explained why the subjects performed the dynamic stability test to anterior (top direction)more easily, since they needed more time to performed movement to posterior (bottom left or bottom right) (Table 3 ) and it can be assumed that patients had less ability to compensate if they fell to the posterior direction.

The point at which all the weight of the body can be concentrated is known as the center of gravity. The center of gravity depends on

Table 4 Correlation Coefficients (Spearman's) between Static and Dynamic Postural Stability and Mobility Function

\begin{tabular}{lcc}
\hline \multicolumn{1}{c}{ Correlation } & p-value & R \\
\hline X with TUG & 0.005 & 0.975 \\
Y with TUG & 0.138 & 0.376 \\
$\begin{array}{l}\text { Postural stability in top right } \\
\text { quarter with TUG }\end{array}$ & 0.067 & 0.706 \\
$\begin{array}{l}\text { Postural stability in top left } \\
\text { quarter with TUG }\end{array}$ & 0.344 & 0.046 \\
$\begin{array}{l}\text { Postural stability in bottom left } \\
\text { quarter with TUG }\end{array}$ & 0.384 & 0.025 \\
$\begin{array}{l}\text { Postural stability in bottom right } \\
\text { quarter with TUG }\end{array}$ & 0.357 & 0.049 \\
$\begin{array}{l}\text { Note: P<0.005=there was significant correlation between two variables; P>0.005=there was not any significant variable } \\
\text { between two variables }\end{array}$
\end{tabular}


the body's shape and mass distribution. To obtain good acceleration of motion, the center of gravity must be within a person's base of supportto maintain stability. ${ }^{9}$ A studypublished by Schmitt et al. ${ }^{4}$ in 2007 demonstrate that knee instability is an important predictor of knee movement strategies in persons with knee OA. In addition, a study by Hsieh et al. ${ }^{5}$ in 2013, indicate that as thepostural stability decreased in patients with knee OA, the impairment increased. Additionally, postural stability have weak association with the components of body functions and structures of patients with knee OA. ${ }^{5}$ This study also indicated that several patients with decreasing postural stability still can perform activities like walking, standing, and other without help. Meaning their body functions was still good.

In a study by Zang W. et al..$^{10}$ symptomatic knee OA is a common and growing condition that limits physical activity and mobility among many older persons. In this study, there were strong correlations between static postural stability with the impairment of mobility. On the other hand, dynamic postural stability has no correlation with impairment of functional mobility. This is shown on Table 4 where the interpretation from the $\mathrm{p}$ value of static postural stability was $<0.005$, meaning that there were correlations between those two variables. In addition, based on the $r$ value $(r=0.975)$, the correlations between those variables were very strong. In contrast, the correlations between dynamic postural stability and impairment mobility were not found ( $p$ value $=>0.005$ ) .

This study also had limitations. This study was conducted on a small number of subjects since some OA patients had refused to be involved in this study due to limitation of theexaminer to give informed consent considering various educational background of the patient. Subjects of this studywere also limited to the Dr. Hasan Sadikin General Hospital only therefore, it might not become a general reference about correlation of TUG test as functional mobility and postural stability.

This study used the non probability sampling method, which means the subjects were patientswho had a knee OA complaint and was examined directly without their complete medical record for considering other variables as confounding variables which are associated with knee $0 A$, such as the severity of OA, age, sex, weight and trauma.

The inclusion criteria for these samples were only for patients who visited the clinic and were diagnosed as $\mathrm{OA}$ in general and agreed to be examined for factors that may cause decreased static and dynamic postural stability in $\mathrm{OA}$ patients that had not been explored yet.

This study also depended on the software with a fixed program, such as the time and direction of movement that wasalready set by this software. Therefore, not all subjects could perform all the tests because of time limitation arranged by this software.

In conclusion, there is correlation between static postural stability and functional mobility in patients with knee OA. In contrast, there is no correlation between dynamic postural stability and functional mobility in patients with knee OA.

A further study is needed to explore more about factors that may contribute to postural stability in OA patients using other tools and a larger number of subjects.

\section{References}

1. Felson DT. Osteoarthritis of the knee. N Engl J Med. 2006;354(8):841-8.

2. Rosenberg AE. Bones, joints, and softtissue tumors. In: Vinay Kumar M, MD, FRCPath, Abul K. Abbas M, Nelson Fausto $\mathrm{M}$, Jon C. Aster M, PhD, editors. pathologic basis of disease. 8 ed. China: Saunders Elsevier; 2010. p. 1205-56.

3. Wallace D, Barr C. The effect of hip bracing on gait in patients with medial knee osteoarthritis. Arthritis. 2012; 2012: 240376.

4. Schmitt LC, Rudolph KS. Influences on knee movement strategies during walking in persons with medial knee osteoarthritis. Arthritis Rheum. 2007;57(6):1018-26.

5. Hsieh RL, Lee WC, Lo MT, Liao WC. Postural stability in patients with nee osteoarthritis: comparison with controls and evaluation of relationships between postural stability scores and international classification of functioning, disability and health components. Arch Phys Med Rehabil. 2013;94(2):340-6.

6. Melzer I, Benjuya N, Kaplanski J. Postural stability in the elderly: a comparison between fallers and non-fallers. Age Ageing. 2004;33(6):602-7.

7. Alencar M, Arantes P, Dias J, Kirkwood R, Pereira L, Dias R. Muscular function and functional mobility of faller and non-faller elderly women with osteoarthritis of the knee. Braz J Med Biol Res. 2007;40(2):27783.

8. Shaheen A, Ayad K. Impact of chronic 
osteoarthritis of knee joint on postural stability and functional mobility in women. Bull Fac Ph Th Cairo Univ. 2008;13(1):31731.

9. Karduna AR. Introduction of biochemical analysis. In: Oatis CA, editor. Kinesiology the mechanis and pathomechanics of human movement. 2nd ed. India: Lippincott Williams and Wilkins; 2009. p. 13-20.
10. Zhang W, Nuki G, Moskowitz R, Abramson $\mathrm{S}$, Altman $\mathrm{R}$, Arden $\mathrm{N}$, et al. OARSI recommendations for the management of hip and knee osteoarthritis: part III: Changes in evidence following systematic cumulative update of research published through January 2009. Osteoarthritis Cartilage. 2010;18(4):476-99. 